\title{
Comparison of Gold Nanoparticles and Iodinated Contrast Media in Radiation Dose Reduction and Contrast Enhancement in Computed Tomography
}

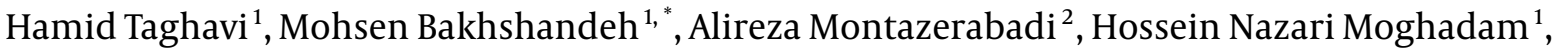 \\ Seyyed Behnam Mazloom Shahri ${ }^{3}$ and Mohammad Keshtkar ${ }^{4, *}$ \\ ${ }^{1}$ Department of Radiology and Radiotherapy, Paramedical Faculty, Shahid Beheshti University of Medical Sciences, Tehran, Iran \\ ${ }^{2}$ Medical Physics Research Center, Mashhad University of Medical Sciences, Mashhad, Iran \\ ${ }^{3}$ Social Determinants of Health Research Center, Gonabad University of Medical Sciences, Gonabad, Iran \\ ${ }^{4}$ Department of Medical Physics and Radiology, Faculty of Medicine, Gonabad University of Medical Sciences, Gonabad, Iran \\ "Corresponding author: Department of Radiology and Radiotherapy, Paramedical Faculty, Shahid Beheshti University of Medical Sciences, Tehran, Iran. Email: \\ mbakhshandeh@sbmu.ac.ir \\ Corresponding author: Department of Medical Physics and Radiology, Gonabad University of Medical Sciences, Gonabad, Iran. Email: keshtkar.dmohammad@yahoo.com \\ Received 2019 April 19; Revised 2019 December 30; Accepted 2020 January 11.
}

\begin{abstract}
Background: Gold nanoparticles with high atomic number and density have good potential to be used as contrast media in computed tomography.

Objectives: In this study, we aimed to assess radiation dose and contrast enhancement performance of gold nanoparticles by measuring contrast to noise ratio compared to clinically used iodinated contrast agents at same concentrations. Contrast enhancement was evaluated in different tube voltages and currents.

Materials and Methods: First, polyethylene glycol coated gold nanoparticles were synthesized with concentrations of $0.5 \mathrm{mM}, 0.6$ $\mathrm{mM}$, and $0.7 \mathrm{mM}$. Gold nanoparticles and iodinated contrast media were scanned with CT imaging system at different tube voltages and time-current product. CT dose index (CTDI) value was measured by special phantom and electrometer. Improving in image contrast was assessed by contrast to noise ratio.

Results: Results showed that gold nanoparticles in all concentrations and energies from 80 to $130 \mathrm{kVp}$ display higher image contrastto-noise ratio (CNR) than iodinated contrast media. Image CNR was increased by increasing $\mathrm{kVp}$ and mAs. The CNR value was maximum at the voltage of 80 and $130 \mathrm{kVp}$ for iodinated compounds and gold nanoparticles, respectively. The CNR value for gold nanoparticles at $130 \mathrm{kVp}$ and $200 \mathrm{mAs}$ was approximately five times higher than that of iodinated compounds.

Conclusion: Gold nanoparticles could be a good candidate for optimizing CT imaging by lowering radiation dose as low as possible while enhancing the image contrast.
\end{abstract}

Keywords: Computed Tomography, Gold Nanoparticles, Iodinated Compounds, Image Contrast, Radiation Dose

\section{Background}

Computed tomography (CT) is one of the most commonly used medical imaging modalities that provides high-resolution three-dimensional (3D) tomography data of the anatomic structures based on different photon attenuation of tissues (1). CT benefits could be derived from easy availability, low cost, and usefulness in tumor detection $(2,3)$. Iodinated compounds with high X-ray absorption coefficient have been utilized for improving CT image contrast for many years (4). However, renal toxicity and speedy clearance by means of the kidney, which provides short imaging times after administration hampered their clinical application $(5,6)$. CT contrast agents based on nanoparticle like polyethylene glycol coated gold nanoparticles have been designed to beat these disadvantages and to produce even higher contrast properties $(7,8)$.

A successful imaging agent must change image signal in order to improve image contrast, have little toxicity and high uptake in the target issue and a prolonged bloodstream circulation time (9).

Nowadays, gold nanoparticles with unique properties can have many of these features as a contrast agent. Biocompatibility with biological tissues and high atomic number and density of gold nanoparticles compared to those of iodine compounds proved its possibility to use 
gold nanoparticles as a contrast agent in CT scan imaging (2).

One of the ways to enhance biocompatibility of gold nanoparticles is to coat them by coating materials such as polyethylene glycol (PEG). Increasing the biocompatibility increases the time nanoparticles spend in the circulatory system, which makes it possible to provide enough time to collect cellular nanoparticles in the vicinity of the cell (1).

There are several studies that proposed gold nanoparticles as a contrast agent for CT. For example, Kim et al. (10), investigated the contrast enhancement of gold nanoparticles and compared them with that of iodinated contrast agent in CT and they reported that gold nanoparticles provide 1.9 times contrast. Jackson et al. (11) reported that gold nanoparticles produce contrast many times greater than iodine based contrast agents.

\section{Objectives}

To our knowledge, no study has discussed applying lower CT imaging factors to reduce radiation dose while maintaining good image quality by using gold nanoparticles as the contrast media. In this study, we aimed to assess contrast enhancement performance of gold nanoparticles by measuring contrast to noise ratio (CNR) compared to clinically used iodinated contrast agents at same concentrations. Contrast enhancement was evaluated in different tube voltages and currents.

\section{Materials and Methods}

\subsection{Gold Nanoparticles Synthesis}

Sixty milligram chloroauric acid $\left(\mathrm{HAuCl}_{4}\right)$ was dissolved in $20 \mathrm{~mL}$ of deionized water and stirred for $15 \mathrm{~min}$ utes at $90^{\circ} \mathrm{C}$. After the solution temperature was fixed, 60 mg polyethylene glycol (2000 MW), 50 milligrams of oleic acid, 50 milligrams of oleylamine and 100 milligrams of sodium citrate were added. Then the resulting solution was stirred for two hours to cool at room temperature (12, 13).

\subsection{Iodine Containing Contrast Media Commonly Used in CT}

Visipaque: Iodixanol is an iso-osmolar, non-ionic and dimeric iodine-containing $x$-ray contrast media with the trade name of Visipaque. Visipaque is available at the concentrations of $150 \mathrm{mg} / \mathrm{mL}, 270 \mathrm{mg} / \mathrm{mL}$ and $320 \mathrm{mg} / \mathrm{mL}$ in glass vials in imaging centers, especially radiology and CT (14).

Meglumine Compound 76\%: Meglumine compound is ionic iodine-containing x-ray contrast media. Each $20 \mathrm{~mL}$ Meglumine compound 76\% contains $7.2 \mathrm{~g}$ iodine (14).

\subsection{Material Characterization}

After synthesizing gold nanoparticles, the concentration of gold nanoparticle solution was determined by ICPOES (Spectro Arcos, Germany). Optical property of gold nanoparticles was evaluated by UV-visible spectrophotometry at wavelengths between $370 \mathrm{~nm}$ to $1000 \mathrm{~nm}$.

\subsection{Phantoms}

For CT dose index (CTDI) measurement, head CTDI phantom (RTI, Sweden) made of acrylic plastic (PMMA) was used. This phantom has $16 \mathrm{~cm}$ diameter and five holes (one in the center and four in the periphery) and $100 \mathrm{~mm}$ length pencil ion chamber would fit in these holes (Figure 1). First, CTDI100 was measured by $100 \mathrm{~mm}$ length pencil ionization chamber in each hole of the CTDI phantom. Then the pencil ionization chamber was inserted in the center (CTDIcenter) hole and at the periphery (CTDIperiphery) holes and weighted CTDI (CTDIW) was calculated by the following equation (15):

$C T D I_{W}=\frac{1}{3}\left(C T D I_{100}\right)_{c e n t e r}+\frac{2}{3}\left(C T D I_{100}\right)_{\text {periphery }}$

Finally, volume CTDI (CTDIv) was calculated with the following relationship (15):

$C T D I_{V}=C T D I_{w} /$ Pitch

Where pitch factor is the ratio of the distance the table travels per revolution to the total nominal beam collimation in spiral imaging mode (15).

For CT imaging, a Plexiglas rectangular phantom was constructed. In this phantom, several places for accommodating $1.5 \mathrm{~mL}$ tube vials were considered (Figure 2).

\subsection{Imaging Protocols}

The gold nanoparticles and iodinated contrast agents were imaged using a 16-slice Somatom Sensation CT scanner (Siemens, Germany). The vials containing gold nanoparticle and iodinated contrast agent samples, all with the same concentration were placed in the imaging phantom.

Phantom containing gold nanoparticle and iodinated contrast agents, all in $0.7 \mathrm{mM}$ concentration, were scanned with a tube voltage of 80,110 and $130 \mathrm{kV}$, and the following tube currents: 50, 80,110,140,170, and $200 \mathrm{~mA}$, using a helical brain protocol. Images were reconstructed using the standard protocol. 

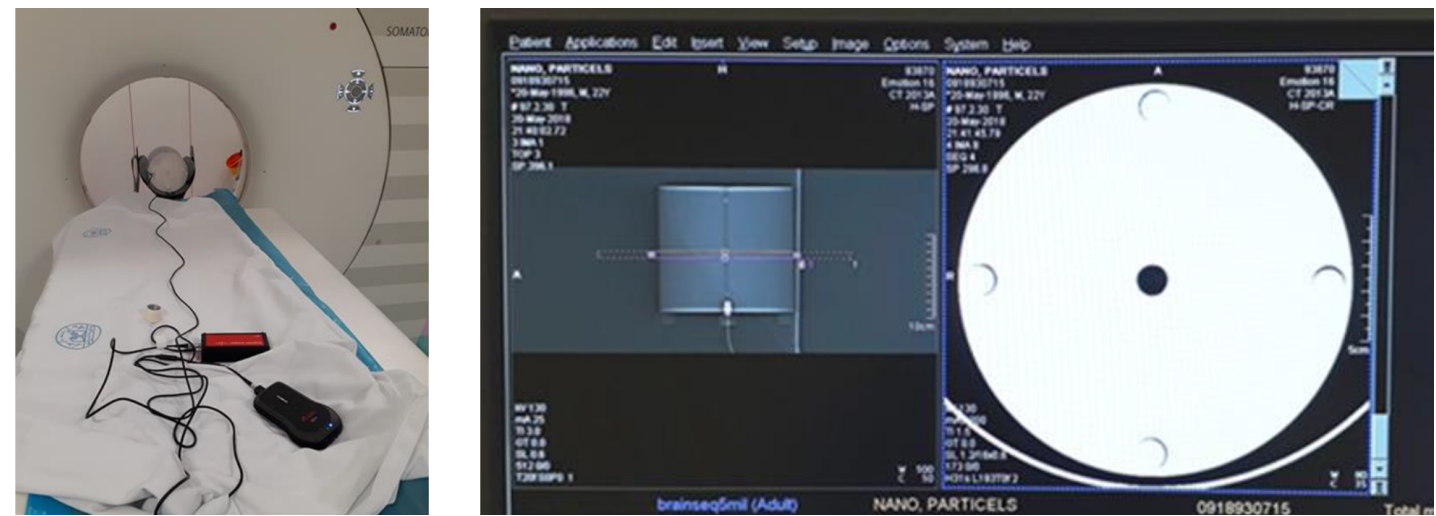

Figure 1. Head CT dose index (CTDI) phantom placed in CT gantry and a $100 \mathrm{~mm}$ ionization chamber inserted in the phantom used for CTDI measurements.

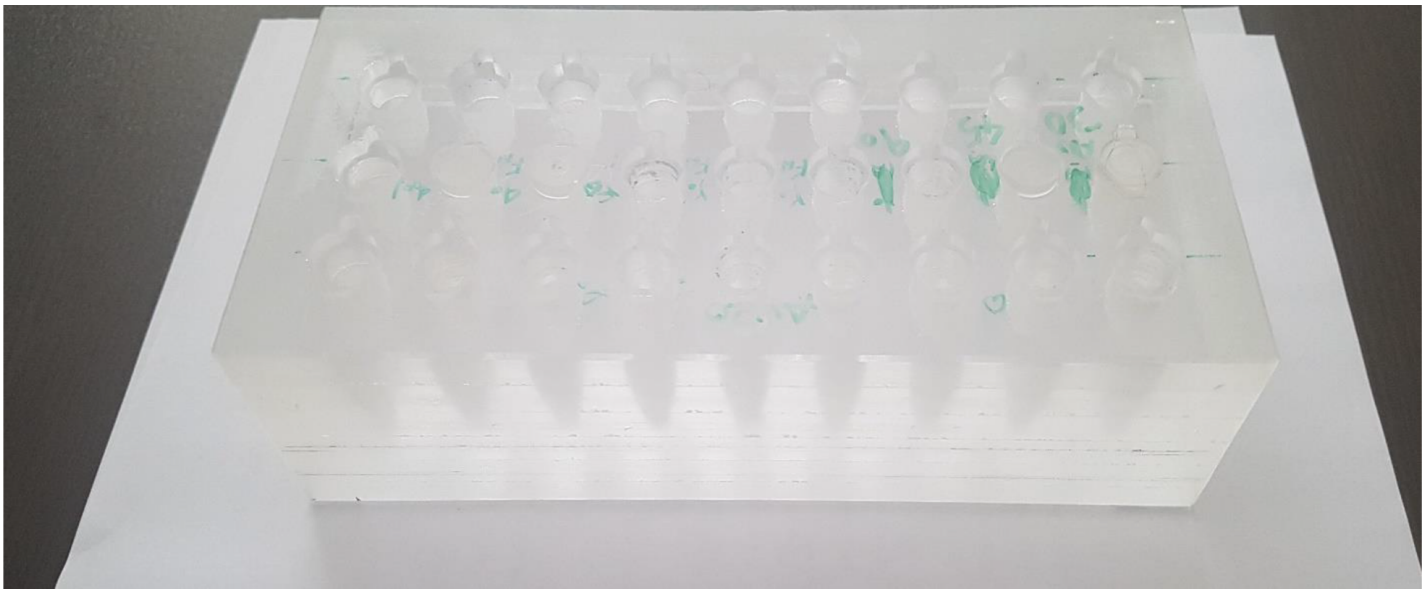

Figure 2. Constructed plexiglas rectangular phantom for imaging gold nanoparticles and iodinated samples

\subsection{Image and Statistical Analysis}

After CT scanning, images were analyzed using syngo software (Siemens, Germany) by putting a region of interest (ROI) in three different slices in every vial and close to the background and getting an attenuation value in Hounsfield units (HU). Means and standard deviations of attenuation values for each vial were recorded and contrast was provided as the difference between the mean attenuation values of the ROI and that of the background. Consequently, CNR values were calculated by dividing contrast to standard deviation of attenuation values in background ROI (16). The variables were tube voltage and contrast media concentration. Statistical analysis was carried out by paired, one tail student's $t$-test method by SPSS software version 22 and a value of $\mathrm{P}<0.05$ was considered statistically significant.

\section{Results}

Figure 3 shows optical absorption of polyethylene gly$\mathrm{col}$ (PEG)-coated gold nanoparticles. It reveals that absorption peak related to them is located at $520 \mathrm{~nm}$.

PEG-coated gold nanoparticles were synthesized in three concentrations of $0.5 \mathrm{mM}, 0.6 \mathrm{mM}$, and $0.7 \mathrm{mM}$. Figure 4A shows an example of CT images of test vials with different concentrations. It can be seen that as the concentration of gold nanoparticles increases, attenuation increases.

Figure $4 \mathrm{~B}$ and $\mathrm{C}$ show images obtained under the same exposure factors at concentrations of $0.6 \mathrm{mM}$ and $0.7 \mathrm{mM}$, respectively. It clearly represents that in similar concentrations, gold nanoparticles show greater attenuation compared to iodinated contrast agents.

Figure 5 shows obtained CNR of gold nanoparticles and iodinated compound images as a function of concentra- 


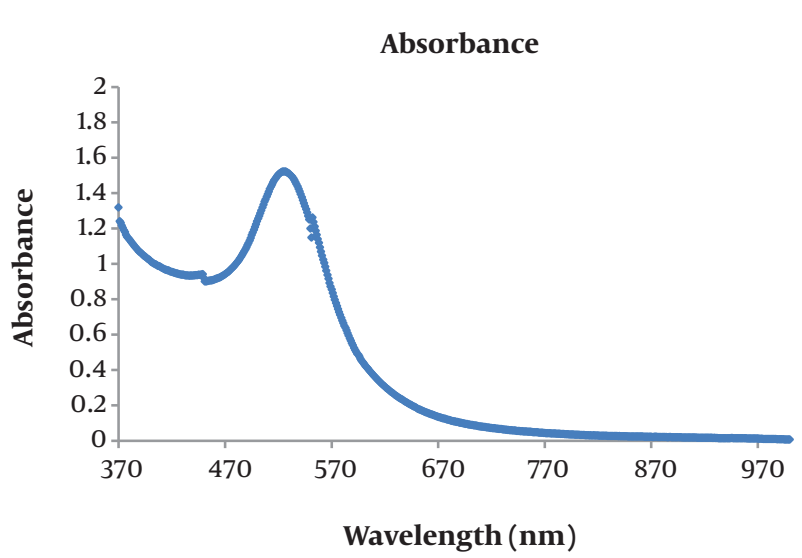

Figure 3. Optical absorption of polyethylene glycol (PEG)-coated gold nanoparticles by UV-Vis spectrum spectrophotometry

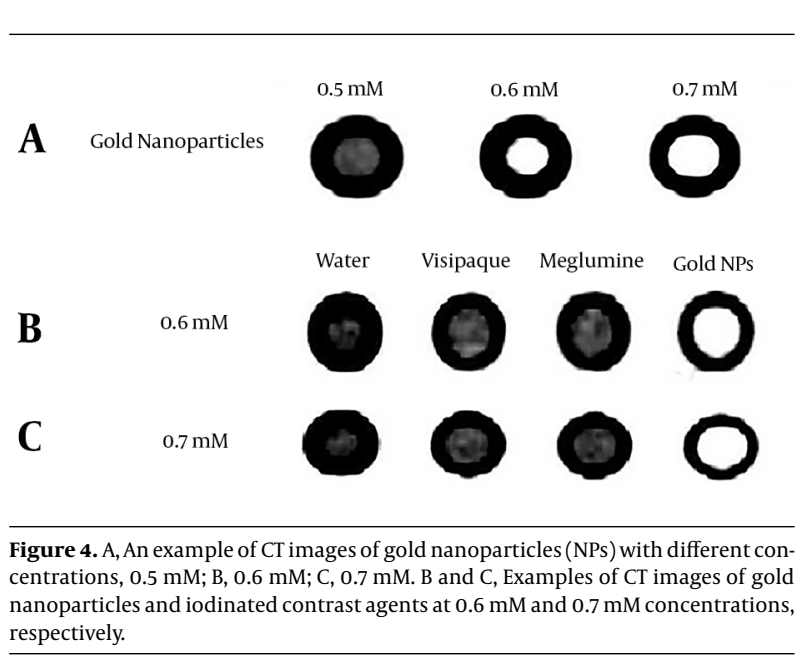

tion. By increasing the concentration of gold nanoparticles and iodinated compound, CNR increases. Increased CNR for gold nanoparticles compared to iodinated compounds was significant for concentrations of 0.6 and 0.7 $\mathrm{mM}(\mathrm{P}<0.05)$.

Figure 6 represents obtained CNR of images as a function of tube potential. By increasing $\mathrm{kVp}, \mathrm{CNR}$ increases for gold nanoparticles, so that they have the highest CNR in 130 $\mathrm{kVp}$. Iodinated compounds have the highest CNR in $80 \mathrm{kVp}$ (K-edge of gold $80.7 \mathrm{keV}$ and iodine $33.2 \mathrm{keV}$ ). Gold nanoparticles compared to iodinated compounds increased CNR significantly for all tube potentials $(\mathrm{P}<0.05)$.

Table 1 shows CNR and CTDI values for gold nanoparticles and iodinated compounds in different $\mathrm{kVp}$ and $\mathrm{mAs}$. CTDI and CNR increases by increasing in mAs and $\mathrm{kVp}$. In each CTDI and concentration of $0.7 \mathrm{mM}$, CNR is significantly higher for gold nanoparticles than iodinated compound $(\mathrm{P}<0.05)$.

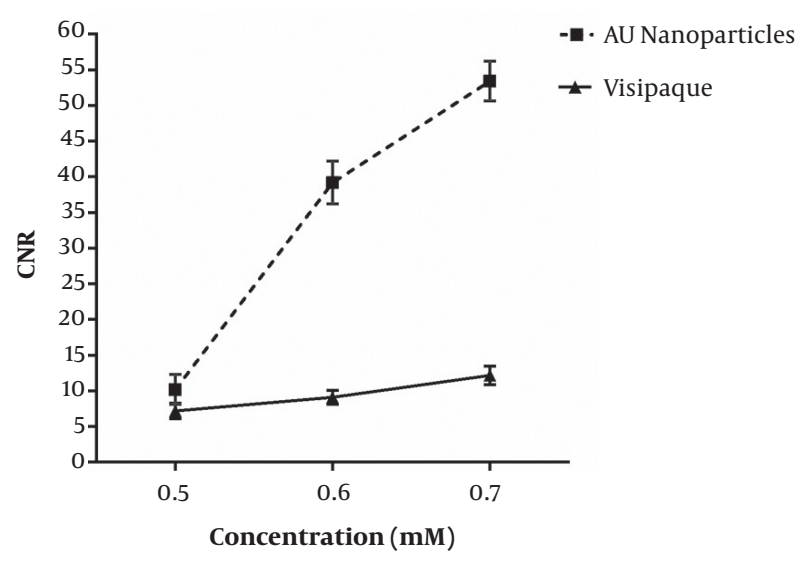

Figure 5. Obtained contrast-to-noise ratio (CNR) of gold nanoparticles and iodinated compound images as a function of concentration (AU, gold)

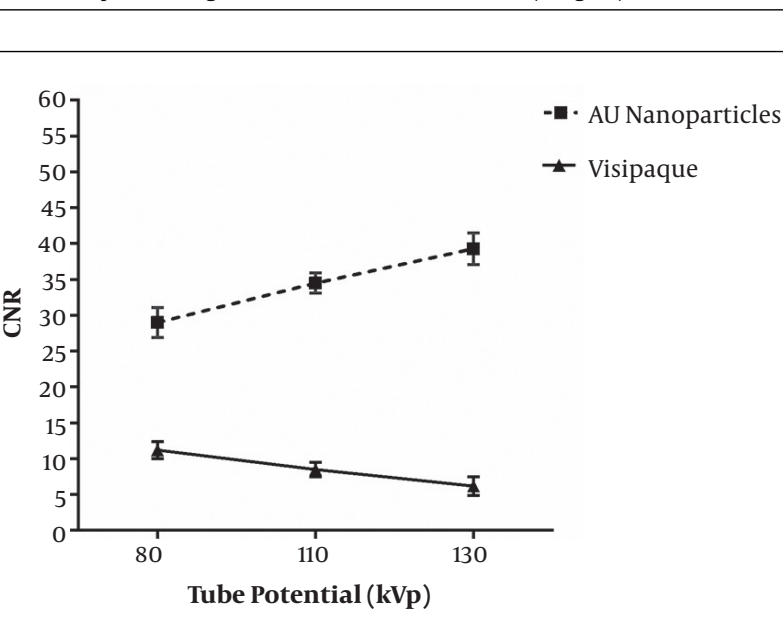

Figure 6. Obtained contrast-to-noise ratio (CNR) of gold nanoparticles and iodinated compound images as a function of tube potential (AU, gold)

\section{Discussion}

In this study, we have shown that gold nanoparticles in concentrations higher than $0.5 \mathrm{mM}$ and energies 80 $130 \mathrm{kVp}$ display higher image CNR than iodinated contrast media. Image CNR for gold nanoparticles increased by increasing $\mathrm{kVp}$ and $\mathrm{mAs}$. The CNR value is maximum for iodinated compounds at voltage of $80 \mathrm{kVp}$ and $200 \mathrm{mAs}$ in the same concentration with gold nanoparticles. The CNR value at $130 \mathrm{kVp}$ and $200 \mathrm{mAs}$ is approximately five times higher than that of iodinated compounds.

Jackson et al. (11) compared the energy dependency of the contrast produced by gold nanoparticles and same concentration of iodinated contrast media. Their results showed that the $\mathrm{kVp}$ has remarkable impact on the contrast enhancement of the two contrast agents. At a concentration of $0.5077 \mathrm{M}$, no significant difference in contrast en- 
Taghavi H et al.

\begin{tabular}{|c|c|c|c|c|}
\hline $\mathbf{k V p}$ & mAs & CNR AUNPs & CNR Visipaque & CTDIv, mGy \\
\hline 130 & 50 & $56 \pm 4.1$ & $7.6 \pm 1$ & 12.05 \\
\hline 130 & 110 & $60.1 \pm 2$ & $8.53 \pm 1.1$ & 26.22 \\
\hline 130 & 200 & $70.8 \pm 5$ & $8.1 \pm 1$ & 47.48 \\
\hline 110 & 110 & $50.3 \pm 1.2$ & $8.1 \pm 0.3$ & 17.96 \\
\hline 110 & 200 & $53.9 \pm 4.1$ & $7.5 \pm 1$ & 32.52 \\
\hline 80 & 50 & $44.1 \pm 3.2$ & $9.6 \pm 1$ & 3.67 \\
\hline 80 & 110 & $44.6 \pm 3.3$ & $14 \pm 2.1$ & 7.98 \\
\hline 80 & 200 & $46.2 \pm 2$ & $17 \pm 1.8$ & 14.45 \\
\hline
\end{tabular}

Abbreviations: AUNPs, gold nanoparticles; CNR, contrast-to-noise ratio; CTDIv, volume CT dose index; SD, standard deviation.

${ }^{\mathrm{a}}$ Values are expressed as mean $\pm \mathrm{SD}$.

hancement was reported at energies routinely utilized for angiography (around $80 \mathrm{kVp}$ ) probably because of the $\mathrm{k}$ edge effect for iodine (11). At the highest tube voltages usually used in computed tomography, CNR for gold nanoparticles was about $114 \%$ greater than that produced by iodine at $140 \mathrm{kVp}$.

In 2012, Galper et al. compared the contrast enhancement of gold nanoparticles with iodinated compounds. They revealed that the contrast enhancement of gold nanoparticles at $120 \mathrm{kVp}$ is 1.9 times higher than that of iodinated compounds (17).

Kim et al. (10) assessed the contrast enhancement of gold nanoparticles with that of iodine contrast agent in the range of 1 to $3 \mathrm{M}$. Their results showed that gold nanoparticles provide 1.9 times better contrast. Our results are consistent with all the above-mentioned studies.

Computed tomography is one of the most important sources of ionizing radiation in medical applications (18). A bedrock of radiation protection is to hold radiation exposure "as low as reasonably achievable" (ALARA principle) (19). ALARA states that the optimal quality of diagnostic image must be obtained at the lowest possible radiation dose. As shown in Table 1, increasing the mAs significantly increases the absorbed dose. However, this increase does not result in noticeable contrast enhancement. At 130 $\mathrm{kVp}$, increasing the tube-current product from 50 to 200 $\mathrm{mAs}$ resulted in approximately 3.8 fold increase in radiation dose, whereas the corresponding contrast enhancement was not noticeable (1.2 fold). In all CTDI values, the contrast enhancement obtained by gold nanoparticles are much higher than that of iodinated contrast media. Therefore, gold nanoparticles would reduce radiation dose in CT examination by lowering related $\mathrm{mAs}$, while enhancing the contrast of CT images.
In summary, this study represents that better contrast enhancement could be provided using gold nanoparticles compared to iodinated contrast media. The CNR values obtained by gold nanoparticles increases by increasing $\mathrm{kVp}$ and mAs. Gold nanoparticles could be a good candidate for optimizing CT imaging by lowering radiation dose as low as possible while enhancing the image contrast. In biological environments, contrast enhancement could be more successful by modifying gold nanoparticles with targeted ligands.

\section{Footnotes}

Authors' Contribution: Study concept and design: Hamid Taghavi and Alireza Montazerabadi. Analysis and interpretation of data: Mohsen Bakhshandeh and Mohammad Keshtkar. Drafting of the manuscript: Hamid Taghavi and Mohammad Keshtkar. Critical revision of the manuscript for important intellectual content: Alireza Montazer Abadi and Hossein Nazari Moghadam. Statistical analysis: Seyyed Behnam Mazloom Shahri

Conflict of Interests: There is no conflict of interest.

Ethical Approval: This article does not contain any studies with human participants or animals.

Funding/Support: This research was supported by Shahid Beheshti University of Medical Sciences (IR.SBMU.RETECH.REC.1396.578).

\section{References}

1. Chou SW, Shau YH, Wu PC, Yang YS, Shieh DB, Chen CC. In vitro and in vivo studies of FePt nanoparticles for dual modal CT/MRI molecular imaging. J Am Chem Soc. 2010;132(38):13270-8. doi: 10.1021/ja1035013. [PubMed: 20572667]. 
2. Huang P, Bao L, Zhang C, Lin J, Luo T, Yang D, et al. Folic acidconjugated silica-modified gold nanorods for X-ray/CT imagingguided dual-mode radiation and photo-thermal therapy. Biomaterials. 2011;32(36):9796-809. doi: 10.1016/j.biomaterials.2011.08.086. [PubMed: 21917309].

3. Keshtkar M, Saba V, Mosleh-Shirazi MA. Application of different methods for reducing radiation dose to breast during MDCT. J Biomed Phys Eng. 2018;8(4):341-6. doi: 10.31661/jbpe.v0i0.981. [PubMed: 30568923]. [PubMed Central: PMC6280114].

4. Krause W. Delivery of diagnostic agents in computed tomography. Adv Drug Deliv Rev. 1999;37(1-3):159-73. doi: 10.1016/s0169409x(98)00105-7. [PubMed: 10837733].

5. Haller C, Hizoh I. The cytotoxicity of iodinated radiocontrast agents on renal cells in vitro. Invest Radiol. 2004;39(3):149-54. doi: 10.1097/01.rli.0000113776.87762.49. [PubMed: 15076007].

6. Hizoh I, Haller C. Radiocontrast-induced renal tubular cell apoptosis: Hypertonic versus oxidative stress. Invest Radiol. 2002;37(8):42834. doi: 10.1097/00004424-200208000-00003. [PubMed: 12138358].

7. Rabin O, Manuel Perez J, Grimm J, Wojtkiewicz G, Weissleder R. An Xray computed tomography imaging agent based on long-circulating bismuth sulphide nanoparticles. Nat Mater. 2006;5(2):118-22. doi: 10.1038/nmat1571. [PubMed: 16444262].

8. Kim JS, Kuk E, Yu KN, Kim JH, Park SJ, Lee HJ, et al. Antimicrobial effects of silver nanoparticles. Nanomedicine. 2007;3(1):95-101. doi: 10.1016/j.nano.2006.12.001. [PubMed: 17379174].

9. Boote E, Fent G, Kattumuri V, Casteel S, Katti K, Chanda N, et al. Gold nanoparticle contrast in a phantom and juvenile swine: models for molecular imaging of human organs using x-ray computed tomography. Acad Radiol. 2010;17(4):410-7. doi: 10.1016/j.acra.2010.01.006. [PubMed: 20207313]. [PubMed Central: PMC2835539].

10. Kim D, Park S, Lee JH, Jeong YY, Jon S. Antibiofouling polymercoated gold nanoparticles as a contrast agent for in vivo X-ray computed tomography imaging. J Am Chem Soc. 2007;129(24):7661-5. doi: 10.1021/ja071471p. [PubMed: 17530850].

11. Jackson PA, Rahman WN, Wong CJ, Ackerly T, Geso M. Potential dependent superiority of gold nanoparticles in comparison to iodinated contrast agents. Eur J Radiol. 2010;75(1):104-9. doi: 10.1016/j.ejrad.2009.03.057. [PubMed: 19406594].

12. Zhang XD, Wu D, Shen X, Chen J, Sun YM, Liu PX, et al. Sizedependent radiosensitization of PEG-coated gold nanoparticles for cancer radiation therapy. Biomaterials. 2012;33(27):6408-19. doi: 10.1016/j.biomaterials.2012.05.047. [PubMed: 22681980].

13. Lenart VM, Gómez SL, Calatayud MP, Goya GRF. Size and shape control of magnetite nanoparticles with a nonselective binding surfactants. Arch Prepr Arch. 2014.

14. Tilly G, Hardouin MJC, Lautrou J. X-ray contrast media. Google Patents; 1977.

15. Seeram E. Computed tomography-e-book: Physical principles, clinical applications, and quality control. USA: Elsevier Health Sciences; 2015.

16. Baker ME, Dong F, Primak A, Obuchowski NA, Einstein D, Gandhi N, et al. Contrast-to-noise ratio and low-contrast object resolution on full- and low-dose MDCT: SAFIRE versus filtered back projection in a low-contrast object phantom and in the liver. AJR Am J Roentgenol. 2012;199(1):8-18. doi: 10.2214/AJR.11.7421. [PubMed: 22733888].

17. Kim J, Chhour P, Hsu J, Litt HI, Ferrari VA, Popovtzer R, et al. Use of nanoparticle contrast agents for cell tracking with computed tomography. Bioconjug Chem. 2017;28(6):1581-97. doi: 10.1021/acs.bioconjchem.7b00194. [PubMed: 28485976]. [PubMed Central: PMC5481820].

18. Brenner DJ, Hall EJ. Computed tomography-an increasing source of radiation exposure. N Engl J Med. 2007;357(22):2277-84. doi: 10.1056/NEJMra072149. [PubMed: 18046031].

19. Okrent D. The safety goals of the U.S. Nuclear Regulatory Commission. Science. 1987;236(4799):296-300. doi: 10.1126/science.3563510. [PubMed: 3563510 ]. 